\title{
Dual-Task Does Not Increase Slip and Fall Risk in Healthy Young and Older Adults during Walking
}

\author{
Rahul Soangra and Thurmon E. Lockhart \\ Locomotion Research Laboratory, School of Biological and Health Systems Engineering, Ira A Fulton Schools of Engineering, \\ Arizona State University, Tempe, AZ, USA
}

Correspondence should be addressed to Thurmon E. Lockhart; thurmon.lockhart@asu.edu

Received 6 September 2016; Revised 16 December 2016; Accepted 28 December 2016; Published 31 January 2017

Academic Editor: Estefanía Peña

Copyright (C) 2017 Rahul Soangra and Thurmon E. Lockhart. This is an open access article distributed under the Creative Commons Attribution License, which permits unrestricted use, distribution, and reproduction in any medium, provided the original work is properly cited.

\begin{abstract}
Dual-task tests can identify gait characteristics peculiar to fallers and nonfallers. Understanding the relationship between gait performance and dual-task related cognitive-motor interference is important for fall prevention. Dual-task adapted changes in gait instability/variability can adversely affect fall risks. Although implicated, it is unclear if healthy participants' fall risks are modified by dual-task walking conditions. Seven healthy young and seven healthy older adults were randomly assigned to normal walking and dual-task walking sessions with a slip perturbation. In the dual-task session, the participants walked and simultaneously counted backwards from a randomly provided number. The results indicate that the gait changes in dual-task walking have no destabilizing effect on gait and slip responses in healthy individuals. We also found that, during dual-tasking, healthy individuals adopted cautious gait mode (CGM) strategy that is characterized by reduced walking speed, shorter step length, increased step width, and reduced heel contact velocity and is likely to be an adaptation to minimize attentional demand and decrease slip and fall risk during limited available attentional resources. Exploring interactions between gait variability and cognitive functions while walking may lead to designing appropriate fall interventions among healthy and patient population with fall risk.
\end{abstract}

\section{Introduction}

Slip-induced fall accidents account for $87 \%$ of hip fractures and are associated with considerable medical cost and human suffering in older adults $[1,2]$. Often, such fractures result in immobility [3] and admissions to skilled nursing facility and sometimes lead to death within one year [4]. Walking is a somewhat complex task associated with higher level cognitive processing such as estimation, planning, and realtime adjustments; specifically, executive function is involved. During walking, numerous sensory (visual, proprioception, and vestibular), conscious inputs and competing objectives (e.g., upright posture versus locomotion) are seamlessly integrated across hierarchical systems, with subtle real-time decisions and adjustments made using cognitive capabilities [5].

In essence, gait performance is affected by the simultaneous performance of dual tasks [6-11]. The dualtask paradigm is commonly used to assess multitasking capabilities. It is presumed that multitasking is influenced by age-related changes in attentional capacities [12] and reducing the abilities to shared processing domains for two concurrent tasks [13]. O'Shea and colleagues [14] suggested that detrimental effects of physical task in the presence of competing attentional task supports a "capacity sharing model" of dual-tasking. According to capacity sharing model, performing two attention demanding tasks reduces the performance of one or both tasks when attentional capacity limit is exceeded [14]. Previous research has demonstrated that dual-task interference results in slower gait speeds $[13,15,16]$, reduced cadence $[16,17]$, shorter stride length $[15,16]$, increased stride duration [16], and longer doublesupport time $[13,18]$. Unwittingly, dual-task performances can potentially identify gait characteristics peculiar to fallers and nonfallers [19].

The influence of attention on gait stability has been studied in numerous patient populations and results consistently show decreased gait velocity and increased gait variability 
in dual-task conditions [11, 20-23]. Apparently, persons with history of falls have more significant gait changes while performing dual task than nonfallers [9, 24-26]. Despite their report, dual task and its association with falls continues to be debated, and there is limited knowledge about how dualtasking influenced slip characteristics. Some studies showed worsening of gait performance while concluding dual-tasking as a predictive of falls $[6,27,28]$ while others have failed to establish any relationship $[29,30]$. One of the studies reported that dual-task related gait changes did not provide any additional information than performance under single task conditions [31]. But these differences may be due to several confounds such as age $[7,9]$ and comorbidities $[10$, 27] and kind of attentional demanding task [11, 32]. Some findings corroborate well with previous investigations that poorer ability of subjects to perform a basic mobility task while carrying a cup of water [6] and the cessation of walking when engaged in conversation [27] are both associated with a four times' risk of fall. Lundin-Ohson et al. reported that dual motor task can differentiate fall prone frail elderly from healthy older adults [27]. Dual-task tests can identify gait characteristics peculiar to fallers and nonfallers [19]. The influence of attention on gait stability has been studied in numerous patient populations and results consistently show decreased gait velocity and increased gait variability in dual-task conditions [11, 20-23]. Fall risk is independent of gait speed but is modulated instead by gait variability [33]. Previous studies demonstrate that gait speed and gait unsteadiness may be dissociated [33-35]. Healthy older adults walk with the same small amount of variability as healthy young adults, even though they walk slower than healthy young adults [36]. However, Springer et al. [37] reported that gait variability increased in older fallers and not in young adults and older nonfallers. They reported that healthy young adults and nonfallers maintain their stable gait in dual-task walking and that there is no evidence of detrimental effects of dual-task activities on gait variability associated with aging. In essence, dual-task paradigm is considered more sensitive to identify fall risk since it widens the gap between fallers and nonfallers [37-39].

To stabilize, healthy people are found to decrease their gait speed [37]. Accordingly, elderly nonfallers were also found to decrease their swing times and their gait speed [37]. This dual-task related decline in walking speed is interpreted as an implicit strategy to avoid loss of balance [8]. Reduction of gait speed among groups represents a coping mechanism to handle the attention demanding challenge of the dual-task activity.

The finding of decrease of gait velocity in dual-task walking is undebated and consistent with most of the studies $[20,21,23,37,40]$. Although dual-task related decline in walking speed is not specific for increased risk of falling, increase of stride time variability is closely associated with the occurrence of falls [33, 41]. There exists association between low stride time variability and efficient executive function in healthy older adults [5] and high stride time variability and impaired executive function in demented older adults [23]. Low stride time variability in healthy older adults is associated with minor involvement of attention in the control of the rhythmic stepping mechanism [11].

Previous research has shown that dual-task related gait changes consisted of increase in number of stops, lateral deviations, steps, and walking time $[6,7,11]$ and increase in stride width, stride length, and stride time variabilities $[7,42]$. Intrasubject variability of kinematic variables is an index of movement consistency or stability of gait performance. However, there exists negative correlation between variability in step width and balance performance of the elderly women [43] and also an increased variability in step length for hospitalized fallers compared with nonfallers [44]. Gabell and Nayak [45] could not find any effects of age on variability in step length and step width while walking. Maruyama and Nagasaki [46] reported that temporal variability in stance phase durations in gait cycle was decreasing function of speed. Increasing the walking speed produced linear increment in step width variability in contrast to step length variability in healthy adults [47]. Gabell and Nayak [45] suggested that variability in step length is determined predominantly by gait patterning mechanism; on the contrary, step width variability is largely determined by balance control mechanism. Similarly, Heitmann et al. found negative correlation between balance performance and variability in step width but not the same for balance performance and step length.

1.1. Objective. Performance of secondary task, that is, dual task, affects certain aspects of gait, but the relationship between gait variability, dual-tasking, and slip and fall risk is not well understood. This study was conducted to better understand the motor control of gait and the relationship between an individual's motor variability and fall risk during dual-tasking walking conditions. Exploring dual-task related gait changes is of particular interest in understanding variability because a strong relationship exists between dual-task related gait changes and the risk of falling in older adults $[6,28,29]$.

The primary objective of this study was to investigate the relationship between dual-task and slip-induced fall risk. As per our knowledge, no previous study has looked into effects of dual-tasking on slip and fall risk. This study involves two groups (young and old individuals) with (known) different slip and fall risk [48]. It was hypothesized that dualtasking while walking would affect gait characteristics and may increase the slip initiation characteristics in the elderly individuals and will negatively influence slip-induced risk. It was also hypothesized that friction demand and trip risk measured using toe clearance will be significantly different for normal walking and dual-task walking.

\section{Methods}

2.1. Participants. The sample size was estimated using power analysis on the results of the published study by focusing on sample sizes that are large enough to determine differences between the velocities during normal walking. Palombaro et al. have determined that minimal clinically important 
TABLE 1: Background characteristics of study participants.

\begin{tabular}{lcccc}
\hline & \multicolumn{3}{c}{ Age group } \\
& Mean & SD & Mean & SD \\
\hline Age [years] & 71.14 & 6.51 & 22.64 & 2.56 \\
Height $[\mathrm{cm}]$ & 174.57 & 10.24 & 170.37 & 9.33 \\
Weight $[\mathrm{Kg}]$ & 78.55 & 18.25 & 69.65 & 15.52 \\
BMI & 25.52 & 4.27 & 23.78 & 4.00 \\
\hline
\end{tabular}

difference (MCID) for habitual gait speed is $0.10 \mathrm{~m} / \mathrm{s}$ [49]. The standard deviation of measurement is $0.10 \mathrm{~m} / \mathrm{s}$ [49]. Therefore, means and standard deviations of velocity in this study were used to compute the required sample size (using JMP, version 7, SAS Institute Inc., Cary, NC, 1989-2007). The required sample size for detecting significant differences in velocity, given $\alpha=0.05$ and power $=0.80$ and small effect size (Cohen's $d$ ) of 0.2 [50], was determined with $n=7$ per group. Seven young and seven old participants were recruited for this study. The younger population consisted of college students of Virginia Tech campus, and older adults were retired people in Blacksburg area. The recruited participants were in a general good health condition, with no recent cardiovascular, respiratory, neurological, and musculoskeletal abnormalities. Only one of the elderly participants (O02) was suffering from chronic obstructive pulmonary disease (COPD). All participants were recruited based on criteria of complete ambulation, without the use of any assistive devices, and ability to rise from a chair without assistance and free of orthopedic injury. This study was approved by the Institutional Review Board (IRB) of Virginia Tech. All participants who participated in this study provided written consent prior to the beginning of data collection. Demographic information for the participants is provided in Table 1.

2.2. Instrumentation. The experiments were conducted on a 15-meter linear walking track, embedded with two force plates (BERTEC \#K80102, Type 45550-08, Bertec Corporation, $\mathrm{OH}$ 43212, USA, and AMTI BP400600 SN6780, Advances Mechanical Technology Inc., Watertown, MA 02472, USA). A six-camera ProReflex system (Qualisys, Gothenburg, Sweden) was used to collect three-dimensional kinematics of posture and gait data in participants. Kinematic data were sampled and recorded at $120 \mathrm{~Hz}$. Ground reaction forces of participants walking over the test surfaces were measured using two force plates and sampled at a rate of $1200 \mathrm{~Hz}$. A sixteen-channel surface electromyography (sEMG) DTS Telemyo system (Noraxon 15770N GreenwayHayden Loop, \#100 Scottsdale, AZ, USA), was used to record the temporal activation of two ankle muscles (gastrocnemius and tibialis anterior) in the both lower extremities during walking.

2.3. Experimental Protocol. All participants were first familiarized with laboratory equipment's and were provided a

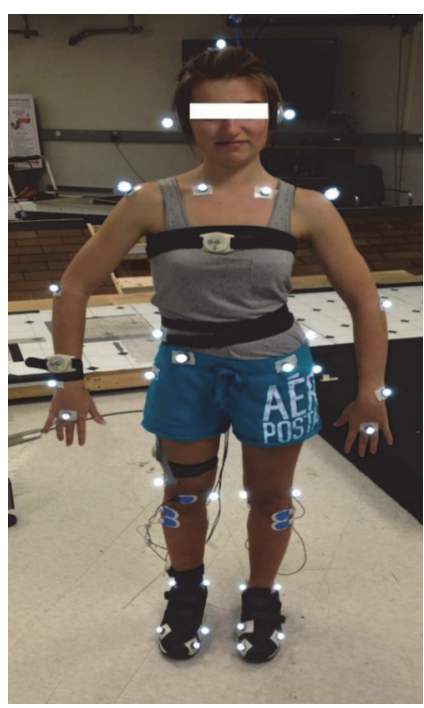

FIGURE 1: The picture of placement of reflective marker, inertial sensors, and s-EMG.

verbal explanation of the experimental procedure. Participants were requested to wear laboratory clothes and shoes, fitting to their sizes. Height and weight of participants were noted below the ID numbers assigned to the subject. Surface Electromyogram (s-EMG) electrodes were affixed by asking participants to plantarflex and dorsiflex their ankle for gastrocnemius and tibialis anterior muscles. Twenty-six reflective markers were attached to various bony landmarks of the body such as head, both ears, both acromioclavicular joints, acromions, humoral condyles, ulnar stylus, knuckles, both right and left anterior superior iliac spine (ASIS), greater trochanters, both medial and lateral condyle of both limbs, malleolus (medial and lateral), and heel and toes of both feet (shown in Figure 1). The marker configuration was similar to that defined by Lockhart et al. 2003 and was used to derive the whole-body center of mass biomechanical model [51]. Kinetic data was acquired using two forceplates such that two consecutive steps would fall on them. The slippery surface (which was at top of the second forceplate) was covered with $1: 1$ water and jelly mixture to reduce the coefficient of friction (COF) of the floor surface (dynamic COF 0.12). Participants were kept unaware of the position of this surface as the embedded forceplates are also covered with similar vinyl texture as the walkway. This is well standardized protocol used in several previous slip and fall research $[48,51]$. The experiment was divided into two sessions: normal session and dual-task session (Figure 2). Each session was separated by 4 days and each participant was randomly assigned to either normal or dual task as his/her first session.

2.3.1. Normal Walking and Slip. After attaching s-EMGs and markers, participants were instructed to walk on the walkway for 15-20 minutes at their self-selected pace. Participant's gait data were acquired using motion capture, IMUs, forceplates, and EMG system. The starting point during the walk was adjusted such that their nonslipping foot (nondominant) 


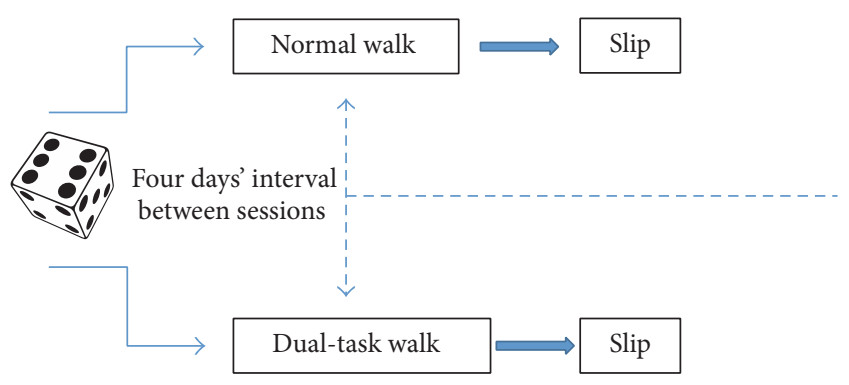

Figure 2: Participants were assigned to normal or dual-task session randomly and the listed tests were conducted.

landed on the first forceplate and dominant foot landed on the second platform. The participants were told in the session that they "may or may not slip," and they should look forward while walking. Additionally, participants were unaware of the placement of slippery surface. Once five walking trials with complete foot fall on the forceplate were obtained, the slippery surface was introduced above the forceplate where dominant foot was expected to strike.

2.3.2. Dual-Task Walking and Slip. This study used a clear and standardized cognitive task, such as serial subtraction $[52,53]$. This session was similar to normal walking session described above, except that the participants were counting backwards when walking. The investigator told a random number before the walking trial and participants had to subtract the number by three continuously until he/she reached the other end of walkway. The investigator corrected the participants, if error was made in counting backwards.

2.4. Data Processing. Normal and dual-task walking trials provided kinematic and kinetic data that was filtered using low-pass Butterworth filter at cut-off frequency of $6 \mathrm{~Hz}$. The EMG data was digitally bandpass filtered at $20-500 \mathrm{~Hz}$. The EMG signals were then rectified and low-pass filtered using Butterworth filter with a $6 \mathrm{~Hz}$ cut-off frequency to create a linear envelope. Heel contact (HC) and toe-off (TO) events were identified from the ground reaction forces with threshold set at 11 Newton (see Abbreviations). The analysis was performed from stance phase ( $\mathrm{HC}$ to $\mathrm{TO}$ ) of nonslipping foot.

2.4.1. Gait Variables. In this study, the gait variables assessed for walking conditions were as follows: (i) Step length (SL): the distance travelled by the participant in one step, it is computed as the anterior-posterior directed distance between ipsilateral limb heel and contralateral limb heel markers at one step. (ii) Step width (SW): It is the mediolateral distance travelled in one step, it is computed as the mediolateral distance between the feet during a step. Double-support time (DST): double support is the time when both feet are on the ground. In one stride, there are two double-support time intervals. It starts with initial contact of one foot until the toe-off of the other foot. (iii) Heel contact velocity (HCV): it is the instantaneous horizontal velocity of the heel at the moment of heel contact. Heel contact is defined as the instant at which the vertical force on the forceplate exceeds $11 \mathrm{~N}$. After processing the heel marker data, HCV was extracted by horizontal heel position at $1 / 120 \mathrm{sec}$ before and after heel contact:

$$
\frac{X_{(i+1)}-X_{(i-1)}}{2 \Delta t}
$$

where $i$ is the frame index at the moment of heel contact. The variables $X_{(i+1)}$ and $X_{(i-1)}$ represent the horizontal heel positions at the frames occurring $1 / 120 \mathrm{sec}$ before and after the instant of heel contact, respectively. The time variable $\Delta t$ is $1 / 120 \mathrm{sec}$.

2.4.2. Slip Propensity Measures. (i) Required coefficient of friction (RCOF): the RCOF is the minimum coefficient of friction which is required between the shoe sole and floor interface to prevent slipping. Thus, if the floor surface and shoe tribology can meet RCOF, walking is possible, whereas if the RCOF is greater than the available friction between the shoe and floor surface, then a slip occurs. The RCOF is defined as the ratio of forward horizontal ground reaction force to vertical ground reaction force, $F_{X} / F_{Z}$. (ii) Transverse coefficient of friction (TCOF): the TCOF was defined as the ratio of lateral ground reaction force component to vertical ground reaction force, $F_{Y} / F_{Z}$. Trip propensity measures: (i) toe clearance: toe clearance is a critical event during midswing of the foot when the foot clearance is achieved with minimum height from the ground surface.

2.4.3. Slip-Severity Parameters. (i) Initial slip distance (SDI): initial slip distance begins after heel contact when the first nonrearward positive acceleration of the foot is identified (Figure 3). This SDI is the distance travelled by the heel from this point of no-rearward positive acceleration (minimum velocity) to the time of the first peak in heel acceleration [51]:

$$
\mathrm{SDI}=\sqrt{\left(X_{2}-X_{1}\right)^{2}+\left(Y_{2}-Y_{1}\right)^{2}} .
$$

(ii) Slip distance II (SDII): slip distance II begins at the slip-point of SDI. Slip stop for SDII is the point at which the first maximum in horizontal heel velocity occurs after the start of SDII. SDI and SDII are used as indices for comparing the severity of slips (Figure 3). (iii) Peak sliding heel velocity (PSHV): it is the maximum forward speed of the heel during slipping. This parameter is calculated using the time derivative of heel marker position during the slip.

(iv) Time2SDI: it was defined as the time to reach midslip from slip start or time to cover SDI. (v) TimeSDI2SDII: it was defined as the time taken from midslip to slip stop. (vi) Time SD total: it was defined as the time taken from slip start to slip stop events.

2.5. Plantar Flexion Muscle Cocontraction. EMG activity was peak normalized within each subject using the ensemble average method during the complete gait cycle [54]. Then, 


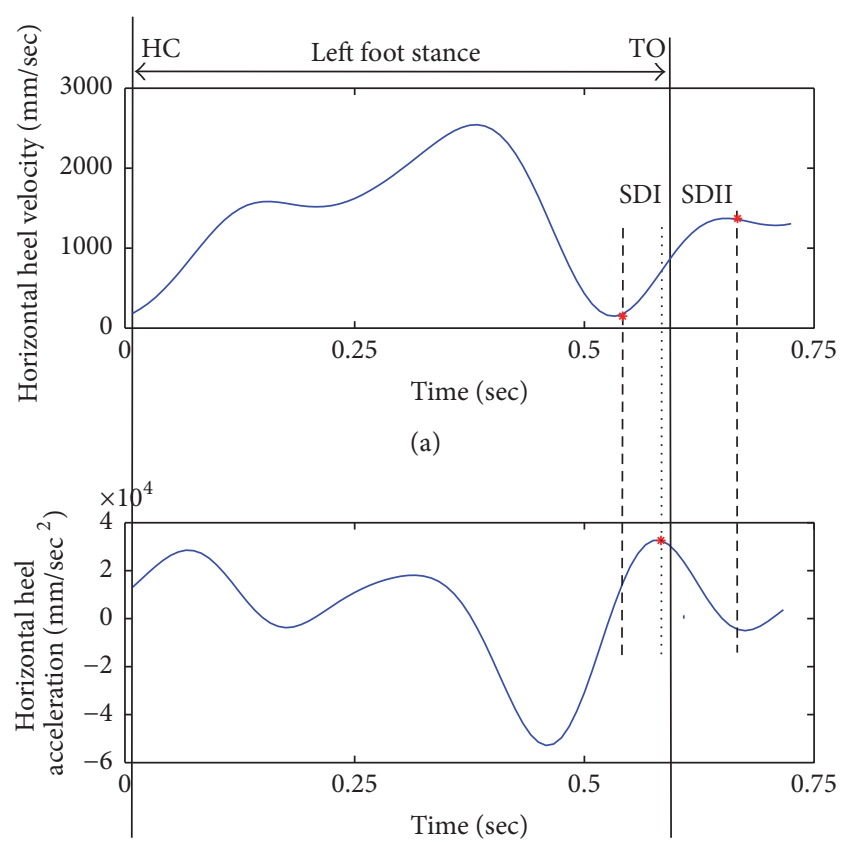

(b)

FIGURE 3: (a) Horizontal heel velocity of slipping foot. (b) Horizontal heel acceleration of the slipping foot.

cocontraction index (CCI) was calculated by the following equation [55]:

$$
\begin{aligned}
\mathrm{CCI}= & \frac{\text { LowerEMG }_{i}}{\text { HigherEMG }_{i}} \\
& \times\left(\text { LowerEMG }_{i}+\text { HigherEMG }_{i}\right),
\end{aligned}
$$

where LowerEMG ${ }_{i}$ refers to the less active muscle at time $i$ and HigherEMG $i$ refers to the more active muscle at time $i$.

The ratio of the EMG activity of tibialis anterior to gastrocnemius was considered for this study (Figure 4). The ratio is multiplied by the sum of activity found in the two muscles. The cocontraction index was defined as the event when bursts of the muscle activity of the agonist and antagonist muscles overlapped for at least $5 \mathrm{~ms}$ [56]. Slipseverity parameters associated with cocontraction indexes among slipping foot (SF) (right foot in all our trials) and contralateral limb (nonslipping foot (NSF) i.e., left foot in all our slip trials): (vii) SFMeanCCIvalue: it was defined as the mean CCI in slipping foot during slip start to slip stop. (viii) SFPeakCCIvalue: it was defined as the peak CCI value during slip start to slip stop. (ix) SFTime2PeakCCIfromNSFHC: it was defined as the time to generate peak ankle cocontraction from the heel contact of unperturbed foot. (x) NSFMeanCCI value: it was defined as the mean CCI in nonslipping foot during slip start to slip stop. (xi) NSFStanceTime: it is the single stance duration in nonslipping foot right before the perturbation event.

2.6. Mini-Mental State Examination (MMSE). The MMSE examines multiple areas of cognition in human brain. The

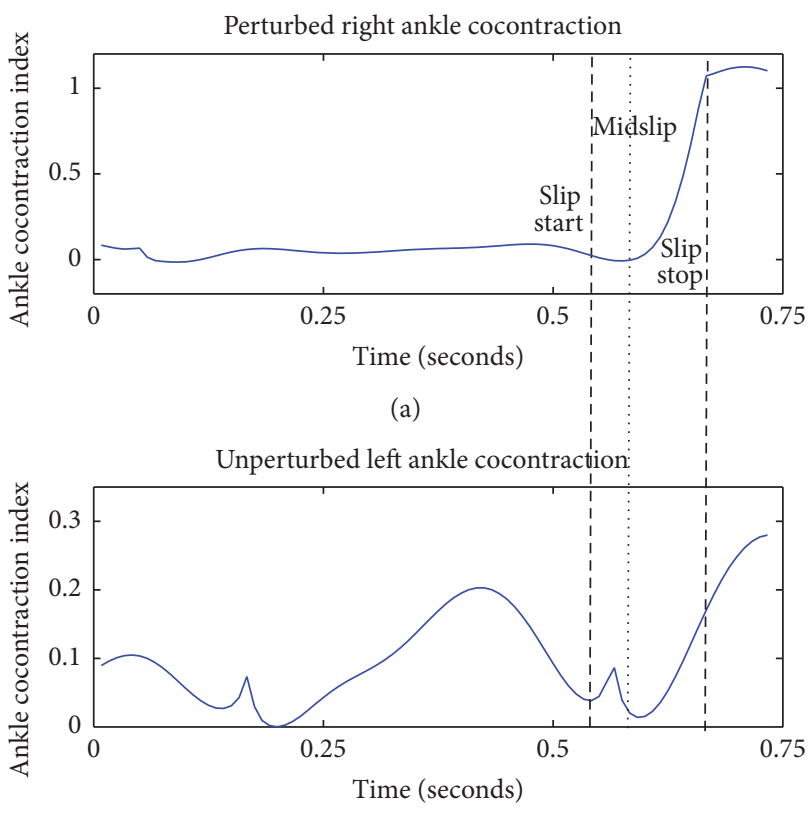

(b)

Figure 4: (a) Ankle cocontraction values in the slipping foot. (b) Ankle cocontraction values in nonslipping foot.

highest possible score is 30; a score of less than 24 denotes cognitive impairment. Mild cognitive impairment is reflected in scores of 18 to 23 , moderate cognitive impairment is suggested by scores of 17 to 10 , and severe cognitive impairment is denoted by scores of less than 10 [57].

2.7. Statistical Design. There were two independent variables: age group (young versus old) and condition (normal versus dual task). Mixed factor multivariate analysis of variance (MANOVA) was conducted where age group was a betweensubjects factor and dual-task/normal conditions group was within-subject factor. Using the Wilks' Lambda test, the MANOVA allowed for determination of which factors had significant effects on the multiple dependent variables as a whole (i.e., gait parameters, muscle cocontraction, and slip parameters). Following MANOVA test, subsequent univariate ANOVA (mixed factor design) were conducted separately for each dependent variable.

All statistical analyses were conducted using JMP (Pro 10.0.2, SAS Institute Inc.) with significance level of $\alpha=$ 0.05 for all the statistical tests. All dependent variables were evaluated for normality (using Shapiro-Wilk $W$ test) and residual analysis. The results did not indicate any violation of normality assumptions.

\section{Results}

3.1. Gait Changes due to Dual-Task Performance. The results indicated that both age groups (young/old) were affected by dual task and their step length ( $\mathrm{df}=1, p<0.0046)$ decreased significantly. Double-support time (DST) $(\mathrm{df}=1$, $p<0.0048)$ and mean single stance time (SST) $(\mathrm{df}=1, p<$ 
TABLE 2: (a) Dual-task changes in gait parameters. (b) General gait parameters for younger and older population for normal and dual-tasking types of walking.

(a)

\begin{tabular}{|c|c|c|c|c|c|}
\hline & \multicolumn{2}{|c|}{ Dual task walk } & \multicolumn{2}{|c|}{ Normal walk } & \multirow{2}{*}{$p$ value } \\
\hline & Mean & SD & Mean & $\mathrm{SD}$ & \\
\hline Step length $[\mathrm{mm}]^{*}$ & 703.79 & 39.25 & 750.89 & 47.87 & 0.004 \\
\hline Step width [mm] & 119.10 & 27.67 & 115.92 & 25.45 & 0.69 \\
\hline $\mathrm{HCV}[\mathrm{mm} / \mathrm{s}]$ & 1191.20 & 765.06 & 1029.41 & 193.01 & 0.55 \\
\hline GCT $[s]$ & 1.12 & 0.06 & 1.08 & 0.06 & 0.075 \\
\hline $\operatorname{DST}[\mathrm{s}]^{*}$ & 0.26 & 0.03 & 0.24 & 0.03 & 0.004 \\
\hline Gait speed $[\mathrm{m} / \mathrm{s}]$ & 1.11 & 0.16 & 1.17 & 0.14 & 0.06 \\
\hline $\operatorname{SST}[s]^{*}$ & 0.70 & 0.04 & 0.66 & 0.05 & 0.013 \\
\hline Step time $[\mathrm{s}]$ & 0.55 & 0.04 & 0.53 & 0.04 & 0.067 \\
\hline Swing time $[\mathrm{s}]$ & 0.43 & 0.03 & 0.42 & 0.02 & 0.54 \\
\hline Toe clearance & 16.40 & 8.29 & 16.29 & 7.62 & 0.96 \\
\hline $\mathrm{RCOF}$ & 0.19 & 0.03 & 0.20 & 0.03 & 0.08 \\
\hline TCOF & 0.07 & 0.02 & 0.07 & 0.01 & 0.90 \\
\hline
\end{tabular}

(b)

\begin{tabular}{|c|c|c|c|c|c|c|c|c|c|c|c|c|}
\hline & \multicolumn{12}{|c|}{ Age group } \\
\hline & \multirow{2}{*}{\multicolumn{6}{|c|}{$\begin{array}{c}\text { Old } \\
\text { Condition }\end{array}$}} & \multirow{2}{*}{\multicolumn{6}{|c|}{$\begin{array}{c}\text { Young } \\
\text { Condition }\end{array}$}} \\
\hline & & & & & & & & & & & & \\
\hline & \multicolumn{3}{|c|}{ DTW } & \multicolumn{3}{|c|}{ NW } & \multicolumn{3}{|c|}{ DTW } & \multicolumn{3}{|c|}{ NW } \\
\hline & Mean & $\mathrm{SD}$ & $\mathrm{CV}$ & Mean & SD & $\mathrm{CV}$ & Mean & $\mathrm{SD}$ & $\mathrm{CV}$ & Mean & $\mathrm{SD}$ & $\mathrm{CV}$ \\
\hline Step length [mm] & 702.92 & 48.75 & 6.94 & 739.10 & 57.89 & 7.83 & 704.26 & 35.36 & 5.02 & 757.24 & 42.76 & 5.65 \\
\hline Step width $[\mathrm{mm}]$ & 117.89 & 21.29 & 18.06 & 113.16 & 20.25 & 17.89 & 119.74 & 31.37 & 26.20 & 117.41 & 28.53 & 24.30 \\
\hline $\mathrm{HCV}[\mathrm{mm} / \mathrm{s}]$ & 993.76 & 516.51 & 51.98 & 1048.44 & 195.34 & 18.63 & 1297.52 & 870.85 & 67.12 & 1019.16 & 198.94 & 19.52 \\
\hline GCT $[s]$ & 1.11 & 0.07 & 6.47 & 1.08 & 0.07 & 6.17 & 1.13 & 0.06 & 5.12 & 1.08 & 0.06 & 5.88 \\
\hline DST [s] & 0.27 & 0.03 & 10.60 & 0.22 & 0.02 & 9.48 & 0.26 & 0.03 & 11.84 & 0.24 & 0.03 & 11.99 \\
\hline SST [s] & 0.68 & 0.05 & 7.11 & 0.65 & 0.04 & 5.72 & 0.71 & 0.04 & 5.85 & 0.66 & 0.05 & 7.62 \\
\hline Step time $[\mathrm{s}]$ & 0.55 & 0.04 & 8.09 & 0.53 & 0.04 & 7.80 & 0.56 & 0.03 & 6.22 & 0.53 & 0.03 & 6.59 \\
\hline Swing time $[\mathrm{s}]$ & 0.42 & 0.02 & 5.71 & 0.43 & 0.03 & 5.90 & 0.44 & 0.03 & 6.60 & 0.42 & 0.02 & 5.54 \\
\hline Toe clearance $[\mathrm{mm}]$ & 18.66 & 12.43 & 66.62 & 19.58 & 5.94 & 30.31 & 15.18 & 5.19 & 34.18 & 14.52 & 8.03 & 55.32 \\
\hline RCOF & 0.17 & 0.02 & 12.92 & 0.19 & 0.02 & 9.05 & 0.19 & 0.03 & 14.25 & 0.20 & 0.03 & 15.24 \\
\hline TCOF & 0.07 & 0.01 & 17.59 & 0.07 & 0.01 & 17.57 & 0.07 & 0.02 & 23.70 & 0.08 & 0.01 & 19.43 \\
\hline Gait speed $[\mathrm{m} / \mathrm{s}]$ & 1.08 & 0.19 & 17.58 & 1.17 & 0.16 & 13.68 & 1.15 & 0.14 & 12.17 & 1.18 & 0.12 & 10.17 \\
\hline
\end{tabular}

${ }^{*} p<0.05$.

0.013 ) increased as well in both young and elderly subjects. It was also found that RCOF and TCOF values decreased slightly due to dual-tasking in both younger and older individuals but the effects were not statistically significant. Older adults were also found to have higher linear variability in some of the gait variables as measured by standard deviation and coefficient of variation of step width, HCV, DST, SST, and gait cycle time due to dual-tasking (as seen in Tables 2(a) and 2(b)).

3.2. Effects of Dual-Tasking Induced Changes in Slip Characteristics. It was found from the variable SFTime2PeakCCIFromNSFHC that the elderly people generated peak ankle muscle cocontractions in half of the time taken by younger adults $(p<0.001)$ (Table 3(a)). Also, in the same line, the results of NSFMeanCCI value also depict that mean coactivity in nonslipping foot during the time of slip start to slip stop is significantly higher in older adults.

It was also found that Time2SDI was significantly increased in dual-task walking trials $(p=0.04)$ although there were no significant differences in SDI (Table 3(b)). Interaction effects were seen for the NSF Mean CCI value ( $p=0.02)$ for the two independent variables, age group and slipping condition (normal versus dual task). It was also found that dual task increased the nonslipping foot stance time $(p=0.03)$ in both young and elderly participants compared to that in normal walk slip condition (Table 3(c)). The MMSE score ranged from 28 to 30 for all older participants, whereas all younger participants scored 30 . 
TABLE 3: (a) Normal slip parameters in young and older individuals. (b) Slip parameters for normal and dual-task conditions. (c) Slip parameters in young and older individuals in normal and dual-task walking condition.

(a)

\begin{tabular}{lccccc}
\hline & & \multicolumn{3}{c}{ Age group } & \multicolumn{2}{c}{ Young } \\
& & Old & SD & CV \\
\hline Time2SDI [s] & Mean & SD & CV & Mean & 0.008 \\
TimeSDI2SDII [s] & 0.046 & 0.014 & 31.492 & 0.051 & 0.032 \\
TimeSDTotal [s] & 0.069 & 0.013 & 18.182 & 0.081 & 39.817 \\
SDI [mm] & 0.115 & 0.024 & 20.889 & 0.132 & 0.034 \\
SDII [mm] & 38.867 & 23.605 & 60.733 & 33.063 & 13.556 \\
SDTotal [mm] & 116.722 & 43.147 & 36.966 & 154.175 & 88.275 \\
SF MeanCCIvalue & 154.713 & 64.122 & 41.446 & 186.863 & 94.645 \\
SF PeakCCIvalue & 0.081 & 0.043 & 53.626 & 0.093 & 51.001 \\
SF Time2PeakCCIFromNSFHC ${ }^{*}[\mathrm{~s}]$ & 0.938 & 0.809 & 86.261 & 0.828 & 0.111 \\
NSF MeanCCIvalue* & 0.383 & 0.412 & 107.398 & 0.752 & 0.396 \\
PHSV & 0.052 & 0.030 & 57.476 & 0.022 & 0.081 \\
NSF StanceTime [s] & 1033.688 & 404.843 & 39.165 & 1062.313 & 0.013 \\
\end{tabular}

(b)

\begin{tabular}{|c|c|c|c|c|c|}
\hline & \multicolumn{2}{|c|}{ Dual-task slip } & \multicolumn{2}{|c|}{ Normal walk slip } & \multirow{2}{*}{$p$ value } \\
\hline & Mean & $\mathrm{SD}$ & Mean & $\mathrm{SD}$ & \\
\hline Time2SDI $[s]^{*}$ & 0.07 & 0.00 & 0.05 & 0.01 & 0.047 \\
\hline TimeSDI2SDII [s] & 0.08 & 0.01 & 0.08 & 0.03 & 1 \\
\hline TimeSDTotal $[\mathrm{s}]$ & 0.14 & 0.01 & 0.13 & 0.03 & 0.46 \\
\hline $\mathrm{SDI}[\mathrm{mm}]$ & 17.65 & 3.89 & 35.00 & 16.65 & 0.19 \\
\hline SDII $[\mathrm{mm}]$ & 87.09 & 20.68 & 141.69 & 76.20 & 0.43 \\
\hline SDTotal $[\mathrm{mm}]$ & 104.20 & 16.38 & 176.15 & 84.10 & 0.34 \\
\hline SF MeanCCIvalue & 0.06 & 0.05 & 0.09 & 0.09 & 0.69 \\
\hline SF PeakCCIvalue & 0.36 & 0.01 & 0.86 & 0.53 & 0.24 \\
\hline SFTime2PeakCCIFromNSFHC [s] & 0.87 & 0.07 & 0.63 & 0.29 & 0.13 \\
\hline NSF MeanCCIvalue & 0.03 & 0.03 & 0.03 & 0.02 & 0.90 \\
\hline PHSV $[\mathrm{mm} / \mathrm{s}]$ & 699.23 & 138.99 & 1052.77 & 332.59 & 0.22 \\
\hline NSF StanceTime $[\mathrm{s}]^{*}$ & 0.75 & 0.02 & 0.65 & 0.05 & 0.03 \\
\hline
\end{tabular}

(c)

\begin{tabular}{|c|c|c|c|c|}
\hline & \multicolumn{4}{|c|}{ Age group } \\
\hline & \multirow{2}{*}{\multicolumn{2}{|c|}{$\begin{array}{c}\text { Old } \\
\text { Slip condition }\end{array}$}} & \multirow{2}{*}{\multicolumn{2}{|c|}{$\begin{array}{c}\text { Young } \\
\text { Slip condition }\end{array}$}} \\
\hline & & & & \\
\hline & DTS & NS & DTS & NS \\
\hline & Mean & Mean & Mean & Mean \\
\hline Time2SDI $[\mathrm{s}]^{*}$ & 0.067 & 0.046 & 0.067 & 0.051 \\
\hline TimeSDI2SDII [s] & 0.083 & 0.069 & 0.067 & 0.081 \\
\hline TimeSDTotal [s] & 0.150 & 0.115 & 0.133 & 0.132 \\
\hline SDI $[\mathrm{mm}]$ & 14.903 & 38.867 & 20.406 & 33.063 \\
\hline SDII [mm] & 101.71 & 116.722 & 72.463 & 154.175 \\
\hline SDTotal [mm] & 115.78 & 154.713 & 92.622 & 186.863 \\
\hline SF MeanCCIvalue & 0.093 & 0.081 & 0.021 & 0.093 \\
\hline SF PeakCCIvalue & 0.368 & 0.938 & 0.355 & 0.828 \\
\hline SFTime2PeakCCIFromNSFHC [s] & 0.917 & 0.383 & 0.817 & 0.752 \\
\hline NSF MeanCCIvalue & 0.011 & 0.052 & 0.059 & 0.022 \\
\hline $\mathrm{PHSV}[\mathrm{mm} / \mathrm{s}]$ & 797.51 & 1033.688 & 600.95 & 1062.313 \\
\hline NSF StanceTime $[\mathrm{s}]^{*}$ & 0.767 & 0.646 & 0.742 & 0.649 \\
\hline
\end{tabular}

${ }^{*} p<0.05$. 


\section{Discussion}

This study examined the effects of dual task on older adults and established a relationship between dual-task adaptations in gait and associated slip and fall risk. Major findings were that the dual-task paradigm influenced slip initiation characteristics by modulating to "safer" or "cautious" gait. Dual-task related gait changes are associated with intrinsic (one's health related) risk factors for falls. As we did not have frail individuals in this study, we found that healthy young and older individuals adapted to dual-task scenarios by shifting to more "cautious" gait. This was well evidenced by a decrease in step length and heel contact velocity and an increase in step width and single and double-support time during gait.

The results suggest that attentional capacity limit for healthy young and old adults is perhaps exceeded during dual-task walking but did not result in instability or increased fall risk. Collectively, the study findings argue in favor of a critical gait behavior: "Preferred speed of walking in healthy human beings requires less allocation of attentional resources for safe transitioning." These findings support previous investigations:

(1) In a seminal work by Lajoie and coworkers, it was found that reaction times when participants were in single support phase were significantly longer than those in double-support phase, suggesting that attentional demands increased with an increase in balance requirement tasks [58]. Thus, attentional demands varied within a gait cycle. Dual-task walking resulted in higher double stance times; thus, it could be inferred that healthy young and old adapt their gait to reduce attentional demand.

(2) Previous research has also reported that dual-task interference results in slower gait speeds $[13,15$, $16]$, reduced cadence $[16,17]$, shorter stride length $[15,16]$, increased stride duration [16], and longer double-support time $[13,18]$. These cautious gait pattern adopted by healthy adults during dual-tasking, characterized by reduced speed, shorter step length, and increased step width, is likely a consequence of adaptations to minimize perturbations to the body and reduce the risk of falls [33] during reduced attentional demands of walking. We also found that heel contact velocity and required coefficient of friction decreased slightly but not statistically significantly during dual-tasking. This unwittingly indicates that several mechanisms contribute to reduce risk of falls and adapt body movements to cautious gait mode, when less attentional resources are available for gait.

Because walking has greater attentional demands, from an information processing viewpoint, walking is not considered an automated task requiring no cognitive processing [58]. Overall analysis of this study suggests that gait in healthy adults was affected by concurrent cognitive tasks and the evidence is sufficiently robust to support the notion of cautious gait. Even in healthy individuals, age-related changes have been reported in cognitive and motor systems; thus, aging

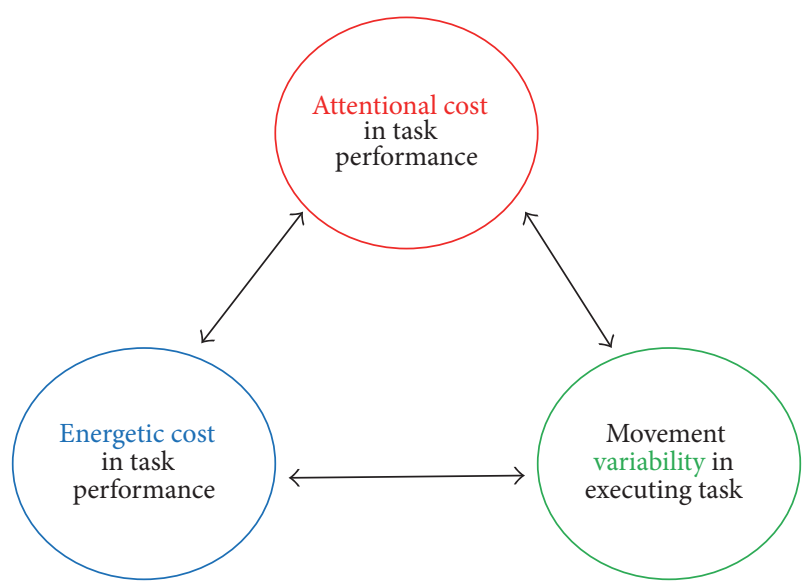

FIGURE 5: Interrelationship of movement variability with attention and energy expenditure while performing task.

may be attributed to higher cognitive-motor interference $[59,60]$. We believe that the dual-task changes observed are compensatory mechanisms to stabilize and allow safe locomotion, in a condition when less attention is available.

Intuitively, the concept of cautious gait adaptations observed in healthy younger and older adults while walking in a dual-task paradigm draws intriguing interests in understanding "Why humans threaten their safety when walking at their preferred speed without dual-tasking?"

Findings of this study elucidate that dual-task related changes in gait do not predispose healthy young and older adults to falls. Healthy people walk at their preferred walking speed, step length, and cadence which is selected to optimize the stability of their gait pattern [27, 61]; this has been addressed in several studies in context of spatial variability $[47,62,63]$ and temporal variability [64]. It is reported that shorter steps and longer double-support times are associated with small sensorimotor and frontoparietal regions, whereas cognitive processing speed is linked to individual differences in gait [65]. Accordingly, Sekiya et al. [47] suggested an optimal method of walking that consists of optimal criteria in terms of energy efficiency, temporal and spatial variability, and attention (Figure 5).

During dual-task walking, less attentional resources are allocated for gait; thus, there is compromise with energy expenditure and variability of kinematic parameters. The present study determined that older adults redressed the diminished attentional investment through differing variability in selective gait parameters including standard deviation and coefficient of variation of step width, HCV, DST, SST, and gait cycle time. Therefore, future studies in energy expenditure with a dual-task criterion would further strengthen intuitive understanding on relationship of energy expenditure, variability, and attention.

This study also evaluated the effects of the dual-task paradigm on slip severity and on linear measures of variability changes in two known healthy age groups. The results suggest that single stance duration is increased in dualtask walking trials which may elicit a congruent adaptation 
in both young and elderly individuals to maintain "stable gait." During the stance phase of a gait cycle, proprioceptive input from extensor muscles and mechanoreceptors in the sole of the foot provide loading information [66] to the central nervous system. Thus, the increased stance duration increases foot-loading information through afferent sensory and proprioceptive mechanoreceptors, such as Golgi-tendon units, muscle spindles, and joint receptors, and may facilitate motor control of the lower extremity during walking [67].

Additionally, the dual-task condition shortened step length significantly, which may be associated with modulation of self-selected pace in order to continue counting rhythmically and need of longer (longer single and double stance) and frequent (shorter steps) proprioception due to dual task [68] or perhaps due to changes in the motor control schema with the adoption of alternative compensatory strategies to increase stability while walking with another task being given primary importance and walking being innately automatic to some extent.

Furthermore, dual-task trials did not significantly affect heel contact velocity (HCV) but slightly decreased RCOF and TCOF in walking trials although these effects were not statistically significant. Considering $\mathrm{HCV}$ as a kinematic gait parameter that can drastically alter the friction demand (by change in required coefficient of friction) [51] and influence the likelihood of slip-induced falls [69-71], dual-task conditions ultimately decrease slip-induced fall risks. Likewise, considering dual-task events had no deleterious effects on toe clearance; therefore, it is inferred that participants delineated reduced trip risk as well.

Through the parameter SF Time2PeakCCIFromLHC, the results suggest that elderly people generated peak ankle muscle cocontractions in half of the time taken by younger adults; that is, they may be quick to introduce ankle muscle coactivity in the slipping foot. Further, coactivity during slipping limits the ankle joint's degrees of freedom, thus reducing requisite motor control adaptability to recover balance after slip. In turn, this affirms that the health status of older age group participating in this study was nonfrail. It should be noted that the subject population recruited for the study was healthy with intact cognitive function (or executive function) with mean Mini-Mental State Examination score above 28 for all.

The NSF Mean CCI value depicted that the mean coactivity in the nonslipping foot during the time of slip start to slip stop was significantly higher in older adults. This phenomenon also lowers the degrees of freedom in the nonslipping foot. The reduction in degrees of freedom in both the right and left feet may influence slip severity amongst older adults. Although greater SDI was reported in the older population, in accordance with previous studies [48, 51], they were not significantly different for the current population. It was found that the Time2SDI in the dual-task paradigm was significantly increased; thus, if elderly individuals have a higher SDI, they require lower heel velocities to cover SDI, thus showing that slower movement of heel from slip start to midslip is seen as an effect of dual-tasking. Probably, this could also be partially explained by higher transitional acceleration of center of mass, in these individuals. Interaction effects were seen for the NSF Mean CCI value for the two independent variables, age group and slipping condition (normal versus dual task), which is interesting because older people have lower nonslipping ankle coactivation (or stiffness reduced) during dual-tasking when compared to normal slipping. On the contrary, younger individuals have higher coactivity in ankle muscles of nonslipping foot during dualactivity. This might be influenced by age-related involvement of attentional resources for the dual-task; perhaps this dual task (counting backwards by subtracting 3) may not be challenging enough to involve higher attentional resources for younger participants.

In sum, this study investigated the effects of attentional interference (induced by dual task) on gait variability and associated fall risk, particularly to understand the following: (a) what is the effect of dual task on spatiotemporal gait parameters? (b) does dual task deteriorate or modify to unsafe gait by predisposing to falls? The findings suggest that in everyday walking tasks with increased attention demands would certainly reduce the resources available for other tasks which may be secondary. But the slow speed, wider step width, and longer double-support time adopted by participants [27] may serve to produce a more safer and stable gait $[72,73]$ and energy-efficient speed of progression $[72,74]$ or probably to maintain certain amount of variability in its kinematics. A cautious gait can be typically marked by moderate slowing, reduced stride length, and mild widening of base-of-support characterized by step width [75]. It is also possible that the kinematic adaptations adopted may serve to reduce the cognitive demand necessary to control the continuous disequilibrium inherent to walking $[58,76]$.

\section{Limitations}

The strength of the conclusions of this study must be tempered by the study's limitations. Although all the participants performed similar kind of dual-task while walking, one's exposure to mathematical background or day-to-day usage of arithmetic operations was a confounding variable in this study. Therefore, the dual task involved in this study may not have required equivalent attentional demand for every subject. The values of toe clearance as found in this study are higher since reflective marker at toe was positioned over laboratory shoes. Thus, toe clearance values are limited due to offset and cannot be compared to existing literature toe clearance values.

\section{Conclusion}

Overall, the current research has contributed knowledge about slip risk in healthy young and older adults and the effects that a dual-task paradigm has on slip initiation characteristics and slip severity. The study results suggest that a dual-task elicits a "cautious gait mode" (CGM) which is an innate adaptive response to counter reduced attention while walking. Attention resources are appropriated for the relevant cognitive task (e.g., counting backwards); therefore, the healthy human response is to adopt a cautious gait mode, which includes a shorter step length and longer stance 
duration, acquiring more proprioceptive information from the ground (or using less attentional resources). The response of CGM is innate for healthy human beings, but in the case of frail elderly persons, who require considerable attention for performing relatively perfunctory gait and postural movements, they may find it challenging to maintain stability.

\section{Abbreviations}

\begin{tabular}{|c|c|}
\hline $\mathrm{HC}:$ & Heel contact \\
\hline TO: & Toe-off \\
\hline SL: & Step length \\
\hline SW: & Step width \\
\hline DST: & Double-support time \\
\hline HCV: & Heel contact velocity \\
\hline RCOF: & $\begin{array}{l}\text { Required coefficient of } \\
\text { friction }\end{array}$ \\
\hline TCOF: & $\begin{array}{l}\text { Transverse coefficient of } \\
\text { friction }\end{array}$ \\
\hline SDI: & Slip distance I \\
\hline SDII: & Slip distance II \\
\hline PSHV: & Peak sliding heel velocity \\
\hline Time2SDI: & Time to cover SDI \\
\hline TimeSDI2SDII: & $\begin{array}{l}\text { Time taken from midslip to } \\
\text { slip stop }\end{array}$ \\
\hline Time SD Total: & $\begin{array}{l}\text { Time taken from slip start } \\
\text { to slip stop }\end{array}$ \\
\hline CCI: & Cocontraction index \\
\hline SF: & Slipping foot \\
\hline NSF: & Nonslipping foot \\
\hline SFMeanCCIvalue: & $\begin{array}{l}\text { Mean CCI in slipping foot } \\
\text { during slip start to slip stop }\end{array}$ \\
\hline SFPeakCCIvalue: & $\begin{array}{l}\text { Peak CCI value during slip } \\
\text { start to slip stop }\end{array}$ \\
\hline SFTime2PeakCCIfromNSFHC: & $\begin{array}{l}\text { Time to generate peak } \\
\text { ankle cocontraction from } \\
\text { the heel contact of } \\
\text { unperturbed foot }\end{array}$ \\
\hline NSFMeanCCI value: & $\begin{array}{l}\text { Mean CCI in nonslipping } \\
\text { foot during slip start to slip } \\
\text { stop }\end{array}$ \\
\hline NSFStanceTime: & $\begin{array}{l}\text { Single stance duration in } \\
\text { nonslipping foot right } \\
\text { before the perturbation } \\
\text { event }\end{array}$ \\
\hline MMSE: & $\begin{array}{l}\text { Mini-Mental State } \\
\text { Examination }\end{array}$ \\
\hline CGM: & Cautious gait mode. \\
\hline
\end{tabular}

\section{Competing Interests}

The authors declare that they have no competing interests.

\section{Acknowledgments}

This research was supported by the NSF-Information and Intelligent Systems (IIS) and Smart and Connected Health (1065442 and 1065262).

\section{References}

[1] D. A. Sterling, J. A. O'Connor, and J. Bonadies, "Geriatric falls: injury severity is high and disproportionate to mechanism," Journal of Trauma-Injury, Infection and Critical Care, vol. 50, no. 1, pp. 116-119, 2001.

[2] W. C. Hayes, E. R. Myers, J. N. Morris, T. N. Gerhart, H. S. Yett, and L. A. Lipsitz, "Impact near the hip dominates fracture risk in elderly nursing home residents who fall," Calcified Tissue International, vol. 52, no. 3, pp. 192-198, 1993.

[3] G. Pioli, F. Lauretani, M. L. Davoli et al., "Older people with hip fracture and IADL disability require earlier surgery," Journals of Gerontology-Series A Biological Sciences and Medical Sciences, vol. 67, no. 11, pp. 1272-1277, 2012.

[4] S. Schnell, S. M. Friedman, D. A. Mendelson, K. W. Bingham, and S. L. Kates, "The 1-year mortality of patients treated in a hip fracture program for elders," Geriatric Orthopaedic Surgery \& Rehabilitation, vol. 1, no. 1, pp. 6-14, 2010.

[5] J. M. Hausdorff, G. Yogev, S. Springer, E. S. Simon, and N. Giladi, "Walking is more like catching than tapping: gait in the elderly as a complex cognitive task," Experimental Brain Research, vol. 164, no. 4, pp. 541-548, 2005.

[6] L. Lundin-Olsson, L. Nyberg, and Y. Gustafson, "'Stops walking when talking' as a predictor of falls in elderly people," The Lancet, vol. 349, no. 9052, p. 617, 1997.

[7] O. Beauchet, R. W. Kressig, B. Najafi, K. Aminian, V. Dubost, and F. Mourey, "Age-related decline of gait control under a dualtask condition," Journal of the American Geriatrics Society, vol. 51, no. 8, pp. 1187-1188, 2003.

[8] V. Dubost, R. W. Kressig, R. Gonthier et al., "Relationships between dual-task related changes in stride velocity and stride time variability in healthy older adults," Human Movement Science, vol. 25, no. 3, pp. 372-382, 2006.

[9] M. Woollacott and A. Shumway-Cook, "Attention and the control of posture and gait: a review of an emerging area of research," Gait and Posture, vol. 16, no. 1, pp. 1-14, 2002.

[10] O. Beauchet, V. Dubost, F. Herrmann, M. Rabilloud, R. Gonthier, and R. W. Kressig, "Relationship between dual-task related gait changes and intrinsic risk factors for falls among transitional frail older adults," Aging Clinical and Experimental Research, vol. 17, no. 4, pp. 270-275, 2005.

[11] O. Beauchet, V. Dubost, K. Aminian, R. Gonthier, and R. W. Kressig, "Dual-task-related gait changes in the elderly: does the type of cognitive task matter?" Journal of Motor Behavior, vol. 37, no. 4, pp. 259-264, 2005.

[12] B. Kerr, S. M. Condon, and L. A. McDonald, "Cognitive spatial processing and the regulation of posture," Journal of Experimental Psychology: Human Perception and Performance, vol. 11, no. 5, pp. 617-622, 1985.

[13] A. Bowen, R. Wenman, J. Mickelborough, J. Foster, E. Hill, and R. Tallis, "Dual-task effects of talking while walking on velocity and balance following a stroke," Age and Ageing, vol. 30, no. 4, pp. 319-323, 2001.

[14] S. O'Shea, M. E. Morris, and R. Iansek, "Dual task interference during gait in people with Parkinson disease: effects of motor versus cognitive secondary tasks," Physical Therapy, vol. 82, no. 9, pp. 888-897, 2002.

[15] D. Hyndman, A. Ashburn, L. Yardley, and E. Stack, "Interference between balance, gait and cognitive task performance among people with stroke living in the community," Disability and Rehabilitation, vol. 28, no. 13-14, pp. 849-856, 2006. 
[16] P. Plummer-D'Amato, L. J. P. Altmann, D. Saracino, E. Fox, A. L. Behrman, and M. Marsiske, "Interactions between cognitive tasks and gait after stroke: a dual task study," Gait and Posture, vol. 27, no. 4, pp. 683-688, 2008.

[17] S. Kemper, J. McDowd, P. Pohl, R. Herman, and S. Jackson, "Revealing language deficits following stroke: the cost of doing two things at once," Aging, Neuropsychology, and Cognition, vol. 13, no. 1, pp. 115-139, 2006.

[18] P. Plummer-D’Amato, L. J. P. Altmann, A. L. Behrman, and M. Marsiske, "Interference between cognition, double-limb support, and swing during gait in community-dwelling individuals poststroke," Neurorehabilitation and Neural Repair, vol. 24, no. 6, pp. 542-549, 2010.

[19] A. Zijlstra, T. Ufkes, D. A. Skelton, L. Lundin-Olsson, and W. Zijlstra, "Do dual tasks have an added value over single tasks for balance assessment in fall prevention programs? A minireview," Gerontology, vol. 54, no. 1, pp. 40-49, 2008.

[20] J. M. Hausdorff, J. Balash, and N. Giladi, "Effects of cognitive challenge on gait variability in patients with Parkinson's disease," Journal of Geriatric Psychiatry and Neurology, vol. 16, no. 1, pp. 53-58, 2003.

[21] G. Yogev, N. Giladi, C. Peretz, S. Springer, E. S. Simon, and J. M. Hausdorff, "Dual tasking, gait rhythmicity, and Parkinson's disease: which aspects of gait are attention demanding?" European Journal of Neuroscience, vol. 22, no. 5, pp. 1248-1256, 2005.

[22] J. M. Hausdorff, J. D. Schaafsma, Y. Balash, A. L. Bartels, T. Gurevich, and N. Giladi, "Impaired regulation of stride variability in Parkinson's disease subjects with freezing of gait," Experimental Brain Research, vol. 149, no. 2, pp. 187-194, 2003.

[23] P. L. Sheridan, J. Solomont, N. Kowall, and J. M. Hausdorff, "Influence of executive function on locomotor function: divided attention increases gait variability in Alzheimer's disease," Journal of the American Geriatrics Society, vol. 51, no. 11, pp. 1633-1637, 2003.

[24] C. Toulotte, A. Thevenon, E. Watelain, and C. Fabre, "Identification of healthy elderly fallers and non-fallers by gait analysis under dual-task conditions," Clinical Rehabilitation, vol. 20, no. 3, pp. 269-276, 2006.

[25] J. Vaillant, P. Martigné, N. Vuillerme et al., "Prediction of falls with performance on Timed 'Up-and-Go' and one-leg-balance tests and additional cognitive tasks," Annales de Readaptation et de Medecine Physique, vol. 49, no. 1, pp. 1-7, 2006.

[26] K. A. Faulkner, M. S. Redfern, J. A. Cauley et al., "Multitasking: association between poorer performance and a history of recurrent falls," Journal of the American Geriatrics Society, vol. 55, no. 4, pp. 570-576, 2007.

[27] L. Lundin-Ohson, R. Lars Nyberg, and Y. Gustafson, "Attention, frailty, and falls: the effect of a manual task on basic mobility," Journal of the American Geriatrics Society, vol. 46, no. 6, pp. 758761, 1998.

[28] J. Verghese, H. Buschke, L. Viola et al., "Validity of divided attention tasks in predicting falls in older individuals: A Preliminary Study," Journal of the American Geriatrics Society, vol. 50, no. 9, pp. 1572-1576, 2002.

[29] B. R. Bloem, J. A. G. Steijns, and B. C. Smits-Engelsman, "An update on falls," Current Opinion in Neurology, vol. 16, no. 1, pp. 15-26, 2003.

[30] P. A. Stalenhoef, J. P. M. Diederiks, J. A. Knottnerus, A. D. M. Kester, and H. F. J. M. Crebolder, "A risk model for the prediction of recurrent falls in community-dwelling elderly: a prospective cohort study," Journal of Clinical Epidemiology, vol. 55, no. 11, pp. 1088-1094, 2002.
[31] A. Bootsma-van der Wiel, J. Gussekloo, A. J. M. De Craen, E. Van Exel, B. R. Bloem, and R. G. J. Westendorp, "Walking and talking as predictors of falls in the general population: The Leiden 85-plus Study," Journal of the American Geriatrics Society, vol. 51, no. 10, pp. 1466-1471, 2003.

[32] G. Allali, R. W. Kressig, F. Assal, F. R. Herrmann, V. Dubost, and $\mathrm{O}$. Beauchet, "Changes in gait while backward counting in demented older adults with frontal lobe dysfunction," Gait and Posture, vol. 26, no. 4, pp. 572-576, 2007.

[33] B. E. Maki, "Gait changes in older adults: predictors of falls or indicators of fear?" Journal of the American Geriatrics Society, vol. 45, no. 3, pp. 313-320, 1997.

[34] J. M. Hausdorff, H. K. Edelberg, S. L. Mitchell, A. L. Goldberger, and J. Y. Wei, "Increased gait unsteadiness in communitydwelling elderly fallers," Archives of Physical Medicine and Rehabilitation, vol. 78, no. 3, pp. 278-283, 1997.

[35] J. M. Hausdorff, A. Lertratanakul, M. E. Cudkowicz, A. L. Peterson, D. Kaliton, and A. L. Goldberger, "Dynamic markers of altered gait rhythm in amyotrophic lateral sclerosis," Journal of Applied Physiology, vol. 88, no. 6, pp. 2045-2053, 2000.

[36] J. M. Hausdorff, S. L. Mitchell, R. Firtion et al., "Altered fractal dynamics of gait: Reduced stride-interval correlations with aging and Huntington's disease," Journal of Applied Physiology, vol. 82, no. 1, pp. 262-269, 1997.

[37] S. Springer, N. Giladi, C. Peretz, G. Yogev, E. S. Simon, and J. M. Hausdorff, "Dual-tasking effects on gait variability: the role of aging, falls, and executive function," Movement Disorders, vol. 21, no. 7, pp. 950-957, 2006.

[38] B. R. Bloem, V. V. Valkenburg, M. Slabbekoorn, and M. D. Willemsen, "The multiple tasks test: development and normal strategies," Gait and Posture, vol. 14, no. 3, pp. 191-202, 2001.

[39] E. W. De Hoon, J. H. Allum, M. G. Carpenter et al., "Quantitative assessment of the stops walking while talking test in the elderly," Archives of Physical Medicine and Rehabilitation, vol. 84, no. 6, pp. 838-842, 2003.

[40] J. H. Hollman, F. M. Kovash, J. J. Kubik, and R. A. Linbo, "Agerelated differences in spatiotemporal markers of gait stability during dual task walking," Gait and Posture, vol. 26, no. 1, pp. 113-119, 2007.

[41] J. M. Hausdorff, D. A. Rios, and H. K. Edelberg, "Gait variability and fall risk in community-living older adults: a 1-year prospective study," Archives of Physical Medicine and Rehabilitation, vol. 82, no. 8, pp. 1050-1056, 2001.

[42] P. C. Grabiner, S. T. Biswas, and M. D. Grabiner, "Age-related changes in spatial and temporal gait variables," Archives of Physical Medicine and Rehabilitation, vol. 82, no. 1, pp. 31-35, 2001.

[43] D. K. Heitmann, M. R. Gossman, S. A. Shaddeau, and J. R. Jackson, "Balance performance and step width in noninstitutionalized, elderly, female fallers and nonfallers," Physical Therapy, vol. 69, no. 11, pp. 923-931, 1989.

[44] R. M. Guimaraes and B. Isaacs, "Characteristics of the gait in old people who fall," International Rehabilitation Medicine, vol. 2, no. 4, pp. 177-180, 1980.

[45] A. Gabell and U. S. L. Nayak, "The effect of age on variability in gait," Journals of Gerontology, vol. 39, no. 6, pp. 662-666, 1984.

[46] H. Maruyama and H. Nagasaki, "Temporal variability in the phase durations during treadmill walking," Human Movement Science, vol. 11, no. 3, pp. 335-348, 1992.

[47] N. Sekiya, H. Nagasaki, H. Ito, and T. Furuna, "Optimal walking in terms of variability in step length," Journal of Orthopaedic and Sports Physical Therapy, vol. 26, no. 5, pp. 266-272, 1997. 
[48] T. E. Lockhart, J. L. Smith, and J. C. Woldstad, "Effects of aging on the biomechanics of slips and falls," Human Factors, vol. 47, no. 4, pp. 708-729, 2005.

[49] K. M. Palombaro, R. L. Craik, K. K. Mangione, and J. D. Tomlinson, "Determining meaningful changes in gait speed after hip fracture," Physical Therapy, vol. 86, no. 6, pp. 809-816, 2006.

[50] C. J. Ferguson, "An effect size primer: a guide for clinicians and researchers," Professional Psychology: Research and Practice, vol. 40, no. 5, pp. 532-538, 2009.

[51] T. E. Lockhart, J. C. Woldstad, and J. L. Smith, "Effects of agerelated gait changes on the biomechanics of slips and falls," Ergonomics, vol. 46, no. 12, pp. 1136-1160, 2003.

[52] E. Strauss, E. M. S. Sherman, and O. Spreen, A Compendium of Neuropsychological Tests: Administration, Norms, and Commentary, Oxford University Press, 2006.

[53] M. D. Lezak, Neuropsychological Assessment, Oxford University Press, Cary, NC, USA, 1995.

[54] M. P. Kadaba, H. K. Ramakrishnan, M. E. Wootten, J. Gainey, G. Gorton, and G. V. B. Cochran, "Repeatability of kinematic, kinetic, and electromyographic data in normal adult gait," Journal of Orthopaedic Research, vol. 7, no. 6, pp. 849-860, 1989.

[55] K. S. Rudolph, M. J. Axe, T. S. Buchanan, J. P. Scholz, and L. Snyder-Mackler, "Dynamic stability in the anterior cruciate ligament deficient knee," Knee Surgery, Sports Traumatology, Arthroscopy, vol. 9, no. 2, pp. 62-71, 2001.

[56] P. Tang and M. Woollacott, "Inefficient postural responses to unexpected slips during walking in older adults," The Journals of Gerontology: Series A, vol. 53, no. 6, p. M471, 1998.

[57] M. F. Folstein, S. E. Folstein, and P. R. McHugh, "'Mini-mental state. A practical method for grading the cognitive state of patients for the clinician," Journal of Psychiatric Research, vol. 12, no. 3, pp. 189-198, 1975.

[58] Y. Lajoie, N. Teasdale, C. Bard, and M. Fleury, "Attentional demands for static and dynamic equilibrium," Experimental Brain Research, vol. 97, no. 1, pp. 139-144, 1993.

[59] J. O. Judge, S. Ounpuu, and R. B. Davis III, "Effects of age on the biomechanics and physiology of gait," Clinics in Geriatric Medicine, vol. 12, no. 4, pp. 659-678, 1996.

[60] R. D. Seidler, J. A. Bernard, T. B. Burutolu et al., "Motor control and aging: links to age-related brain structural, functional, and biochemical effects," Neuroscience and Biobehavioral Reviews, vol. 34, no. 5, pp. 721-733, 2010.

[61] M. D. Latt, H. B. Menz, V. S. Fung, and S. R. Lord, "Walking speed, cadence and step length are selected to optimize the stability of head and pelvis accelerations," Experimental Brain Research, vol. 184, no. 2, pp. 201-209, 2008.

[62] M. Yamasaki, T. Sasaki, and M. Torii, "Sex difference in the pattern of lower limb movement during treadmill walking," European Journal of Applied Physiology and Occupational Physiology, vol. 62, no. 2, pp. 99-103, 1991.

[63] J. S. Brach, R. Berthold, R. Craik, J. M. VanSwearingen, and A. B. Newman, "Gait variability in community-dwelling older adults," Journal of the American Geriatrics Society, vol. 49, no. 12, pp. 1646-1650, 2001.

[64] S. Frenkel-Toledo, N. Giladi, C. Peretz, T. Herman, L. Gruendlinger, and J. M. Hausdorff, "Effect of gait speed on gait rhythmicity in Parkinson's disease: variability of stride time and swing time respond differently," Journal of NeuroEngineering and Rehabilitation, vol. 2, article 23, 2005.
[65] C. Rosano, H. Aizenstein, J. Brach, A. Longenberger, S. Studenski, and A. B. Newman, "Gait measures indicate underlying focal gray matter atrophy in the brain of older adults," Journals of Gerontology - Series A Biological Sciences and Medical Sciences, vol. 63, no. 12, pp. 1380-1388, 2008.

[66] V. Dietz and J. Duysens, "Significance of load receptor input during locomotion: a review," Gait \& Posture, vol. 11, no. 2, pp. 102-110, 2000.

[67] J. Zhang, T. E. Lockhart, and R. Soangra, "Classifying lower extremity muscle fatigue during walking using machine learning and inertial sensors," Annals of Biomedical Engineering, vol. 42, no. 3, pp. 600-612, 2014.

[68] B. A. Smith, M. Kubo, and B. D. Ulrich, "Gait parameter adjustments for walking on a treadmill at preferred, slower, and faster speeds in older adults with down syndrome," Current Gerontology and Geriatrics Research, vol. 2012, Article ID 782671, 7 pages, 2012.

[69] G. M. Karst, P. A. Hageman, T. F. Jones, and S. H. Bunner, "Reliability of foot trajectory measures within and between testing sessions," Journals of Gerontology-Series A Biological Sciences and Medical Sciences, vol. 54, no. 7, pp. M343-M347, 1999.

[70] P. M. Mills and R. S. Barrett, "Swing phase mechanics of healthy young and elderly men," Human Movement Science, vol. 20, no. 4-5, pp. 427-446, 2001.

[71] P. Parijat and T. E. Lockhart, "Effects of lower extremity muscle fatigue on the outcomes of slip-induced falls," Ergonomics, vol. 51, no. 12, pp. 1873-1884, 2008.

[72] A.-M. Ferrandez, J. Pailhous, and M. Durup, "Slowness in elderly gait," Experimental Aging Research, vol. 16, no. 2, pp. 7989, 1990.

[73] D. A. Winter, A. E. Patla, J. S. Frank, and S. E. Walt, "Biomechanical walking pattern changes in the fit and healthy elderly," Physical Therapy, vol. 70, no. 6, pp. 340-347, 1990.

[74] D. D. Larish, P. E. Martin, and M. Mungiole, "Characteristic patterns of gait in the healthy old," Annals of the New York Academy of Sciences, vol. 515, pp. 18-32, 1988.

[75] J. G. Nutt, "Classification of gait and balance disorders," Advances in Neurology, vol. 87, pp. 135-141, 2001.

[76] Y. Lajoie, N. Teasdale, C. Bard, and M. Fleury, "Upright standing and gait: are there changes in attentional requirements related to normal aging?" Experimental Aging Research, vol. 22, no. 2, pp. 185-198, 1996. 


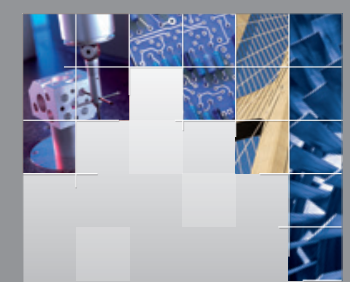

\section{Enfincering}
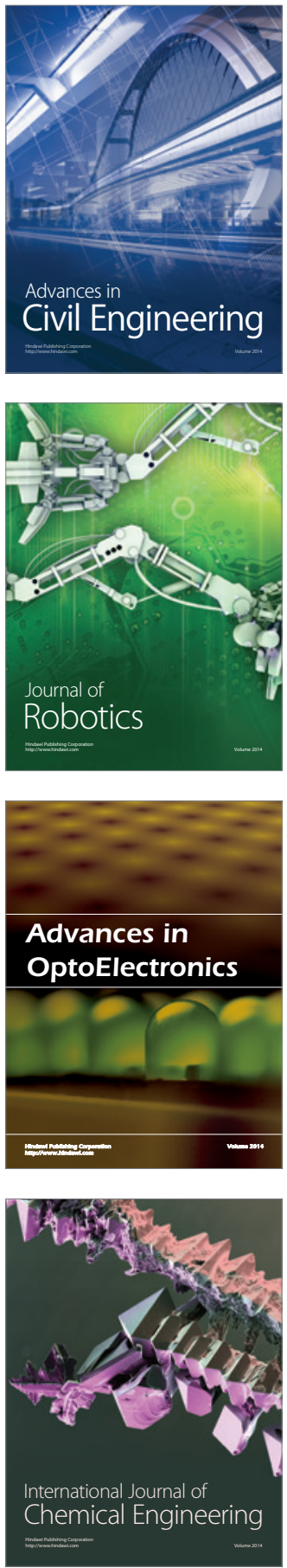

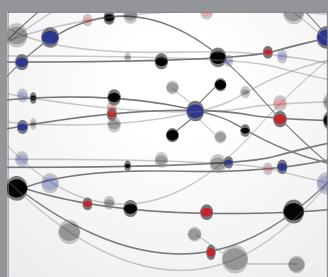

The Scientific World Journal

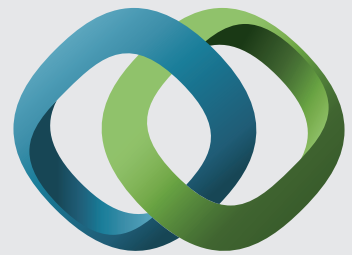

\section{Hindawi}

Submit your manuscripts at

https://www.hindawi.com
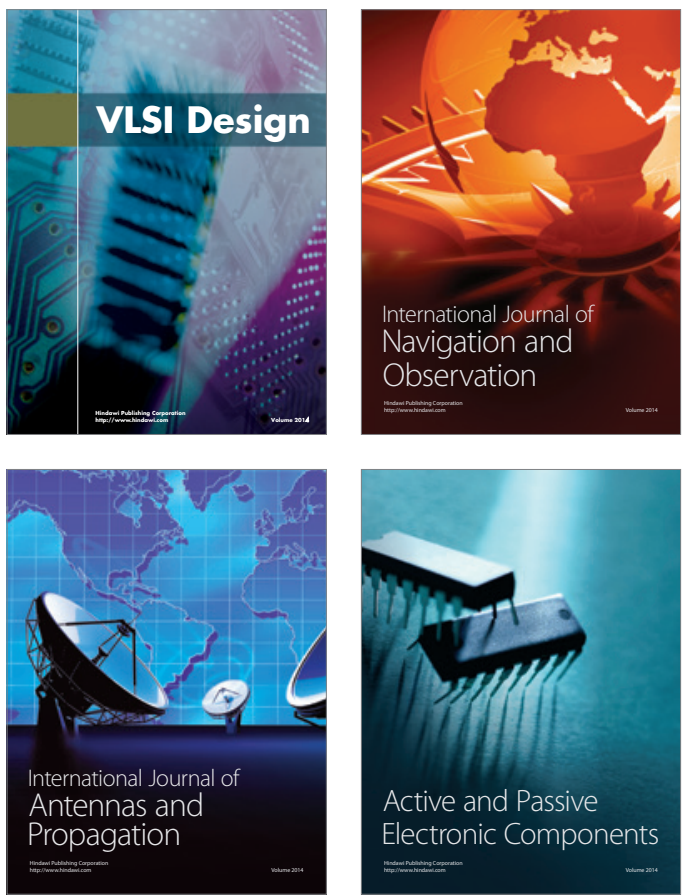
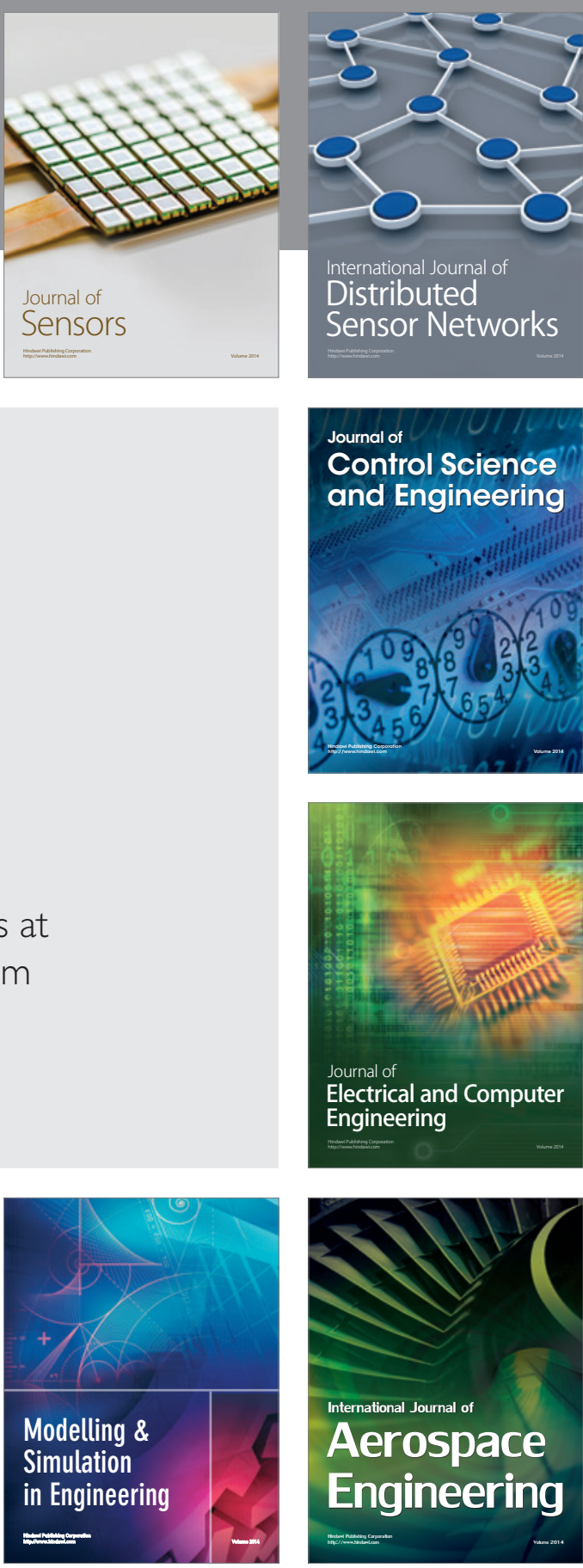

International Journal of

Distributed

Sensor Networks

$-$

Joumal of

Control Science

and Engineering
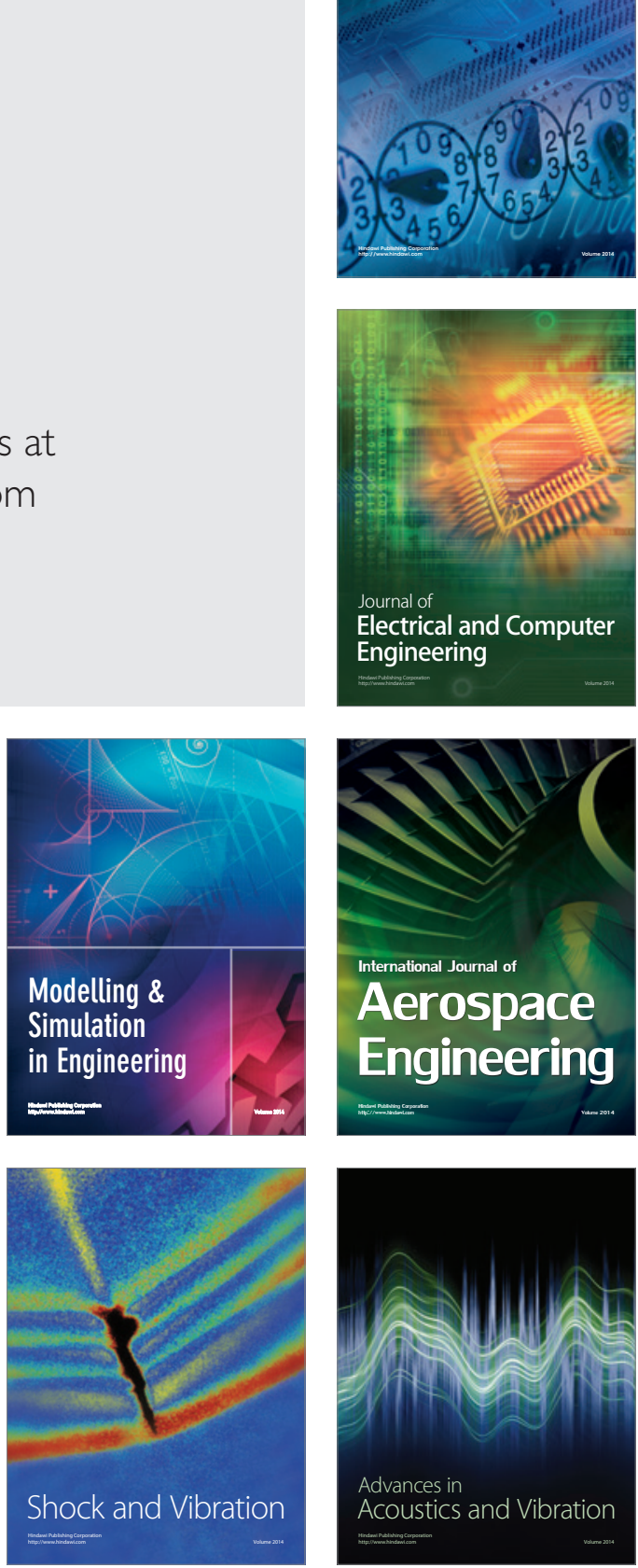